YITP/U-93-29

LBL-34887

\title{
Chaotic Inflation and Baryogenesis in Supergravity*
}

\author{
Hitoshi Murayamal \\ Theoretical Physics Group, Lawrence Berkeley Laboratory \\ University of California, Berkeley, CA 94720, USA \\ Hiroshi Suzukit \\ Instituto Nazionale di Fisica Nucleare, Sezione di Genova, 16146 Genova, Italia \\ T. Yanagida \\ Department of Physics, Tohoku University, Sendai, 980 Japan \\ Jun'ichi Yokoyama \\ Uji Research Center, Yukawa Institute for Theoretical Physics \\ Kyoto University, Uji, 611 Japan
}

\begin{abstract}
We propose a Kähler potential in supergravity which successfully accommodates chaotic inflation. This model can have a large gravitino mass without giving a large mass to squarks and sleptons, and thus is free from both the gravitino problem and entropy crisis. In this model baryogenesis takes place naturally, identifying the inflaton with a right-handed sneutrino with its mass $M \simeq 10^{13} \mathrm{GeV}$, which is consistent with the COBE data and the MikheyevSmirnov-Wolfenstein solution to the solar neutrino problem. The model can also accommodate the matter content appropriate for the mixed dark matter scenario.
\end{abstract}

*This work was supported in part by the Japanese Grant-in-Aid for Scientific Research Fund of Ministry of Education, Science, and Culture, Nos. 05740276 and 05218206, and in part by the Director, Office of Energy Research, Office of High Energy and Nuclear Physics, Division of High Energy Physics of the U.S. Department of Energy under Contract DE-AC03-76SF00098. 


\section{Disclaimer}

This document was prepared as an account of work sponsored by the United States Government. Neither the United States Government nor any agency thereof, nor The Regents of the University of California, nor any of their employees, makes any warranty, express or implied, or assumes any legal liability or responsibility for the accuracy, completeness, or usefulness of any information, apparatus, product, or process disclosed, or represents that its use would not infringe privately owned rights. Reference herein to any specific commercial products process, or service by its trade name, trademark, manufacturer, or otherwise, does not necessarily constitute or imply its endorsement, recommendation, or favoring by the United States Government or any agency thereof, or The Regents of the University of California. The views and opinions of authors expressed herein do not necessarily state or reflect those of the United States Government or any agency thereof of The Regents of the University of California and shall not be used for advertising or product endorsement purposes.

Lawrence Berkeley Laboratory is an equal opportunity employer. 
Cosmic inflation is regarded as a necessary ingredient in the evolution of the early universe not only to solve the horizon and the flatness puzzles [1] as well as the monopole [2], the domain-structure [3] and the gravitino problems [4] but also to account for the origin of density fluctuations [5] as observed by the Cosmic Background Explorer (COBE) satellite 6]. Among the various types of the inflationary models proposed so far, the chaotic inflation [7] is the simplest mechanism to realize the accelerated expansion, which can accommodate inflation even in the presence of large quantum fluctuation in the very early universe.

On the other hand, the idea of inflation generally depends on the existence of the extremely high energy scale, typically of the order of grand-unification scale or Planck scale. This introduces a huge hierarchy between the weak scale and the high energy scale, and the Higgs potential in the standard model becomes unstable against the radiative corrections. The only solution to ensure the stability of the hierarchy in the scalar potential against the radiative corrections is the supersymmetry (SUSY) [8]. Furthermore in chaotic inflation, one has to deal with the scalar fields beyond $M_{P l}$, where supergravity effects will be important.

Unfortunately, these two ideas, chaotic inflation and supergravity, have not been naturally realized simultaneously so far. The main reason is that "minimal" supergravity potential has an expotenential factor which prevents any scalar fields from acquiring values larger than $M_{P l}$ even in the chaotic epoch.

Furthermore, the supergravity model suffers from other difficulties and constraints as listed below. When one adopts the SUSY breaking in the hidden sector, one usually encounters a light scalar field with mass of the order of the gravitino mass $m_{3 / 2}$, and its value at the potential minimum is $O\left(M_{P l}\right)$. It rolls down to the minimum only after $H \sim m_{3 / 2}$, and produces huge entropy with a dilution factor of $O\left(10^{15}\right)$ [9]. It was also recently pointed out that the phenomenon is general in a wide class of superstring inspired models [10. Unlike the gravitino problem, inflation provides no help to solve such an entropy crisis. Of course, we should also have a vanishing cosmological constant at the potential minimum and be able to accommodate $\mathrm{TeV}$ scale squarks/sleptons masses.

In the present letter, we propose a Kähler potential as a model of chaotic inflation in supergravity in which all the problems cited above are resolved. We will also discuss that the inflaton can be identified with the right-handed sneutrino, which was proposed in our previous publication [11]. This idenfication allows us to generate baryon asymmetry in the reheating process, and can naturally accommodate the mixed dark matter scenario.

Before proceeding to our potential, in order to confirm the difficulty in implementing the chaotic inflation into supergravity as mentioned above, let us recall the Lagrangian for scalar multiplets $\varphi^{i}$ in generic supergravity models,

$$
\mathcal{L}_{\varphi}=G_{i}^{j} \partial_{\mu} \varphi^{i} \partial^{\mu} \varphi_{j}^{*}-e^{G}\left[G_{i}\left(G_{j}^{i}\right)^{-1} G^{j}-3\right]
$$

where $G_{i}=\partial G / \partial \varphi^{i}, G^{j}=\partial G / \partial \varphi_{j}^{*}$, and we have taken a unit with $M_{P l} / \sqrt{8 \pi}=1$. In the "minimal" supergravity, to ensure the canonical kinetic term for $\varphi$, one takes the Kähler potential as $G\left[\varphi, \varphi^{*}\right]=\varphi^{i} \varphi_{i}^{*}+\ln |W[\varphi]|^{2}$, where $W[\varphi]$ is the superpotential. Then the scalar potential has an exponential factor $e^{\varphi_{i}^{*} \varphi^{i}}$ which makes it very difficult to incorporate chaotic inflation. In fact, as suggested by Goncharov and Linde, one must introduce either a rather specific class of nonpolynomial superpotential [12] or some fields with nonminimal kinetic term [13. In the former model, one has to arrange the parameters so that the potential is bounded from below while it does not grow rapidly to enable chaotic inflation. It also 
has the same entropy crisis as in the minimal supergravity. We have also found that the latter model [13] suffers from a runaway behavior of the inflaton, since the imaginary part of the inflaton has negative mass squared when the real part is of order of the Planck mass. Then the imaginary part grows without bound, and the scalar potential for the matter fields damps exponentially leading to a vanishing potential.

Now we propose the following Kähler potential free from such difficulties,

$$
\begin{aligned}
G & =\frac{3}{8} \ln \eta+\eta^{2}+\ln |W(\varphi)|^{2}, \\
\eta & =z+z^{*}+\varphi_{i}^{*} \varphi^{i}
\end{aligned}
$$

where $\varphi^{i}$ denotes chiral fields in the model. Then the scalar Lagrangian is given by [14]

$$
\mathcal{L}=\frac{16 \eta^{2}-3}{32 \eta^{2}}\left[\left(\partial_{\mu} \eta\right)^{2}+\left(I_{\mu}\right)^{2}\right]+\frac{16 \eta^{2}+3}{8 \eta}\left|\partial_{\mu} \varphi^{i}\right|^{2}-V
$$

with the potential

$$
\begin{aligned}
V= & \eta^{3 / 8} e^{\eta^{2}}\left\{\frac{8 \eta}{16 \eta^{2}+3}\left|W_{i}\right|^{2}+\frac{\left(16 \eta^{2}-9\right)^{2}}{8\left(16 \eta^{2}-3\right)}|W|^{2}\right\} \\
& +D \text {-terms }
\end{aligned}
$$

where $W_{i}=\partial W / \partial \varphi^{i}$, and $I_{\mu}$ is a $U(1)$ current defined by $I_{\mu}=i \partial_{\mu}\left(z-z^{*}\right)+i\left(\varphi^{i} \partial_{\mu} \varphi_{i}^{*}-\right.$ $\left.\varphi_{i}^{*} \partial_{\mu} \varphi^{i}\right)$

Figure 1 depicts the shape of the potential along the $\eta$-axis. The kinetic term of $\eta$ has the correct signature for $|\eta|>\sqrt{3} / 4$. The minimum of the potential $V$ for $\eta>\sqrt{3} / 4$ is given by $\eta=3 / 4$ and $W_{i}=0$, where the cosmological constant vanishes as desired. Note that the potential Eq. (5) has exactly the same form with the global SUSY case once $\eta$ has settled to its minimum. Therefore, this Kähler potential realizes chaotic inflation, once one has a sensible model within global SUSY. On the other hand, this scalar potential also has flat directions, which might be cosmologically harmful. We return to this point later.

The SUSY is broken down by adding a constant term in the superpotential. The gravitino mass is given by

$$
m_{3 / 2}^{2}=e^{G}=\eta^{3 / 8} e^{\eta^{2}}|W|^{2}
$$

In order to give the gravitino a mass of $m_{3 / 2}$ at the potential minimum, we simply add a constant of $O\left(m_{3 / 2}\right)$ in the superpotential. Note that the constant does not induce the soft SUSY breaking terms to $\varphi^{i}$. Therefore, one can choose the constant freely without giving large masses to the squarks and sleptons. On the other hand, one can give gaugino mass $m_{\lambda}$, assuming the form of the kinetic function $f_{a b}=\delta_{a b} h(z)$,

$$
m_{\lambda}=m_{3 / 2} \frac{\eta\left(16 \eta^{2}+3\right)}{2\left(16 \eta^{2}-3\right)} \frac{\partial h / \partial z}{\Re e(h(z))}=\left.\frac{3}{4} m_{3 / 2} \frac{\partial h / \partial z}{\Re e(h(z))}\right|_{z=\frac{3}{8}}
$$

at the potential minimum $\eta=3 / 4$. The denominator $\Re e(h(3 / 8))$ should be $O(1)$ to give correct gauge coupling constants, while one can choose its derivative in the numerator to 
set gaugino mass below $10 \mathrm{TeV}$ so that radiative corrections do not induce too large masses to squarks and sleptons [15].

Now let us turn to cosmological consideration. It is evident that inflation is possible only in the domain with $\eta>\sqrt{3} / 4$ initially, which we shall assume hereafter. Recall the criterion by Linde [7] that the scalar fields may have any initial configurations at the Planck time as far as the energy density is less than $O(1)$ in the Planck unit. We expect that the initial amplitude of the gauge non-singlet fields at the Planckian epoch are of order unity due to the existence of the $D$-term potential [16]. Also, $\eta$ has $O(1)$ fluctuations from its minimum at that epoch. A gauge-singlet field $\phi$ can have, on the other hand, a value larger than $O(1)$ if its potential $\left|W_{i}\right|^{2}$ is sufficiently flat, and it can drive chaotic inflation. The simplest possibility is

$$
W=\frac{1}{2} M \phi^{2}
$$

where the mass $M$ should be $M \simeq 10^{13} \mathrm{GeV}$ to reproduce COBE data [17]. Then it can have a large value $\phi(0) \simeq 1 / \sqrt{M}$ even though other fields have $O(1)$ value, limited by the last term $|W|^{2}$ in the potential. Then $\eta$ quickly settles to its minimum to make $|W|^{2}$ term practically vanishing, and the potential for the $\phi$ is nothing but the global SUSY potential, $M^{2}|\phi|^{2}$. The other gauge non-singlet fields may drop either to the origin, or one of the flat directions with $O(1)$ amptlidues at most.

The dynamics of the universe after its energy is dominated by that of $\phi$ is the same as in the ordinary chaotic inflation scenario. During the inflation, $\phi(t)$ and the scale factor $a(t)$ are given by

$$
\begin{aligned}
& \phi(t)=\phi(0)-\frac{M}{\sqrt{3}} t \\
& a(t)=a(0) \exp \left[\frac{M}{\sqrt{3}} \phi(0) t\left(1-\frac{M t}{2 \sqrt{3} \phi(0)}\right)\right],
\end{aligned}
$$

respectively. At the time $t_{e}$, when $\phi\left(t_{e}\right) \simeq 1$, the exponential expansion ends. Assuming the initial condition $\phi(0) \simeq 1 / \sqrt{M}$, the duration of the inflation is $t_{e} \simeq 1 / M^{3 / 2}$, and the $e$-folding is $1 / M \simeq 10^{5}$. The necessary initial condition to solve the horizon and flatness problems is actually only $\phi(0) \simeq 15$.

Now we discuss the possible entropy production in our model. The potentially most dangerous field is $\eta$. This field is a superpartner of the goldstino, and therefore one may worry about the huge entropy production [9, 10. Though the true minimum is at $\eta=3 / 4$, the minimum for $\eta$ depends on the values of $|W|^{2}$ and $\left|W_{i}\right|^{2}$ during the inflation. The Fig. 2 shows the minimum of $\eta$ as a function of $\left|W_{i}\right|^{2} /|W|^{2}$. The minimum of $\eta$ was at the true minimum in the beginning of the inflation, but it becomes gradually shifted to $\eta_{e} \simeq 0.70$ at the end of the inflation. It stays there until the expansion rate becomes as small as its mass. If we assume its mass around $O(1) \mathrm{TeV}$, it may produce enormous amount of entropy of $O\left(10^{15}\right)$ dilution factor as mentioned in the beginning. Fortunately, our model allows us to have much larger gravitino mass $m_{3 / 2}$, and simultaneously also for $\eta$, without giving large mass to squarks/sleptons. Then the decay of the coherent oscillation of $\eta$ produces a dilution factor of $\left(\Gamma_{\phi} / \Gamma_{\eta}\right)^{1 / 2}\left(\eta_{e}-3 / 4\right)^{2} / 3$, which is $O(1)$ unless $\Gamma_{\eta} \simeq m_{3 / 2}^{3} \ll 10^{-6} \Gamma_{\phi}$. Therefore our model can avoid the entropy crisis by taking an appropriate particle-physics model of the 
inflaton $\phi$. We will return to this point later. Moreover the gravitinos heavier than $10^{8} \mathrm{GeV}$ decay well before the electroweak phase transition, and are completely harmless; there is no gravitino problem at all [18].

Another possible source of entropy is the flat direction in the SUSY potential whose mass is only of the order of $\mathrm{TeV}$, and the scalar fields may grow along that direction by the quantum fluctuation during the de Sitter expansion of the universe [19]. Though the duration of the inflation, $t_{e} \simeq M^{-3 / 2}$, is long enough to solve horizon and flatness problems, it is too short for the flat direction to grow beyond the Planck scale. The growth of the flat direction field, $\chi$, by the quantum fluctuation is known to be $\left\langle\chi^{2}\right\rangle=\left(H^{3} / 4 \pi^{2}\right) t_{e}$ in the de Sitter space with constant expansion rate $H$. In our model, the exponential expansion begins when the potential is dominated by $M^{2}|\phi|^{2}$ term with $\phi \lesssim 1 / \sqrt{M}$, and the maximum expansion rate is $H \lesssim \sqrt{M}$. Therefore, the amplitude of quantum fluctuations is much smaller than unity; $\sqrt{\left\langle\chi^{2}\right\rangle} \lesssim 1 /(4 \sqrt{3} \pi) \ll 1$. Thus the only fate of the flat direction is to decay with the decay rate $\Gamma \simeq \alpha m(m / \chi)^{1 / 2}$ [20] giving a dilution factor of $O\left(10^{2}\right)$ at maximum even if $\chi=O(1)$ classically at the outset [21].

Finally, we will comment below that this model can successfully incorporate baryogenesis if one identifies $\phi$ with the right-handed sneutrino $\tilde{N}$ [11]. The right-handed neutrino is naturally introduced from particle-physics point of view to account for a small neutrino mass required for the Mikheyev-Smirnov-Wolfenstein (MSW) solution [22] to the solar neutrino puzzle [23] by the seesaw mechanism [24]. Since it is a gauge-singlet field with small Yukawa coupling at least for the first generation, the sneutrino can naturally drive chaotic inflation. The mass $M \simeq 10^{13} \mathrm{GeV}$ which was necessary to reproduce COBE data nicely fits into the window for the MSW solution (see, e.g., 25]). It can also account for baryogenesis quite naturally by first producing lepton asymmetry [26] in the inflaton decay and later converting it partially to baryon asymmetry through the anomalous electroweak process 226 28]. The entropy crisis can be also avoided when its Yukawa coupling is $\lesssim O\left(10^{-5}\right)\left(\Gamma_{N} \lesssim 10^{2} \mathrm{GeV}\right)$ and $m_{3 / 2} \gtrsim 10^{11} \mathrm{GeV}\left(\Gamma_{\eta} \gtrsim 10^{-3} \mathrm{GeV}\right)$.

The resulting baryon-to-entropy ratio $Y_{B}$ is [11]

$$
Y_{B}=0.35 \epsilon \frac{3 T_{R H}}{4 M} \exp \left[-0.012 \frac{G_{F}^{2} m_{\nu_{\tau}}^{2}}{\sin ^{4} \beta} M_{P l} T_{R H}\right],
$$

where $T_{R H}=O\left(10^{9}-10^{10}\right) \mathrm{GeV}$ is the reheating temperature in this model, $\epsilon$ the CPviolating decay asymmetry into leptons and anti-leptons, $G_{F}$ the Fermi constant, $m_{\nu_{\tau}}$ mass of the $\tau$-neutrino, and $\beta$ is the vacuum angle $\tan \beta=\left\langle H_{u}\right\rangle /\left\langle H_{d}\right\rangle$. The exponential factor comes from the annihilation of the lepton numbers in the thermal bath. To account for the desired $Y_{B}$ in the nucleosynthesis, one can take small $\epsilon \simeq 10^{-8}$ with $m_{\nu_{\tau}} \lesssim 2 \mathrm{eV}$, but it requires small $\mathrm{CP}$-violating phase in the Yukawa coupling matrix. More interesting case is that with $O(1)$ CP-violating phase; e.g., $\epsilon \simeq 10^{-3}, T_{R H} \sim 10^{9} \mathrm{GeV}$, and $m_{\nu_{\tau}} \simeq 10 \mathrm{eV}$ [29] gives desired $Y_{B}$ in accordance with the nucleosynthesis. This means that our model does not only accommodate the material ingredients for the mixed dark matter model [30] but in fact it prefers that, with the cold dark matter component consisting of the lightest superparticle. We also mention that the above mechanism is the only intrinsic source of baryogenesis in our model since the flat direction cannot give rise to $B-L$ asymmetry by the Affleck-Dine mechanism [31] in the presence of the large amplitude of $\tilde{N}$ [11]. 
JY acknowledges support by the Research Aid of Inoue Foundation for Science. This work was supported in part by the Japanese Grant-in-Aid for Scientific Research Fund of Ministry of Education, Science, and Culture, Nos. 05740276 and 05218206 . HM acknowledges support by the Director, Office of Energy Research, Office of High Energy and Nuclear Physics, Division of High Energy Physics of the U.S. Department of Energy under Contract DEAC03-76SF00098. 


\section{REFERENCES}

$\S \quad$ On leave of absence from Department of Physics, Tohoku University, Sendai 980, Japan

$\dagger \quad$ On leave of absense from Department of Physics, Ibaraki University, Mito 310, Japan

[1] A.H. Guth, Phys. Rev. D23, 347 (1981).

[2] K. Sato, Mon. Not. R. astr. Soc. 195, 467 (1981); M.B. Einborn and K. Sato, Nucl. Phys. B180 [FS2], 385 (1981).

[3] K. Sato, Phys. Lett. B 99, 66 (1981).

[4] S. Weinberg, Phys. Rev. Lett. 48, 1303 (1982).

[5] S.W. Hawking, Phys. Lett. B 115, 295 (1982); A.A. Starobinsky, Phys. Lett. B 117, 175 (1982); A.H. Guth and S-Y. Pi, Phys. Rev. Lett. 49, 1110 (1982).

[6] G.F. Smoot et al., Astrophys. J. Lett. 396, L1 (1992).

[7] A.D. Linde, Phys. Lett. B 129, 177 (1983).

[8] M. Veltman, Acta Phys. Pol. B12, 437 (1981); L. Maiani, in Proceedings of the Eleventh Gif-sur-Yvette Summer School on Particle Physics, Gif-sur-Yvette, France, 1979 (Inst. Nat. Phys. Nucl. Phys. Particules, Paris, 1980), p.3; S. Dimopoulos and S. Raby Nucl. Phys. B192, 353 (1981); E. Witten, Nucl. Phys. B188, 513 (1981); M. Dine, W. Fischler and M. Srednicki, Nucl. Phys. B189, 575 (1981).

[9] G.D. Coughlan, W. Fishler, E.W. Kolb, S. Raby, and G.G. Ross, Phys. Lett. 131B, 59 (1983).

[10] T. Banks, D.B. Kaplan, and A.E. Nelson, UCSD/PTH 93-26; B. de Carlos, J.A. Casas, F. Quevedo, and E. Roulet, CERN-TH.6958/93.

[11] H. Murayama, H. Suzuki, T. Yanagida, and J. Yokoyama, Phys. Rev. Lett. 70, 1912 (1993).

[12] A.S. Goncharov and A.D. Linde, Phys. Lett. B 139, 27 (1984).

[13] A.S. Goncharov and A.D. Linde, Class. Quantum Grav. 1, L75 (1984).

[14] G. Gelmini, C. Kounnas, and D.V. Nanopoulos, Nucl. Phys. B250, 177 (1985).

[15] In the present model gaugino loops give rise to masses for squarks and sleptons, as in the no-scale model (see, e.g., A.B. Lahanas and D.V. Nanopoulos, Phys. Rept. 145, 1 (1987)).

[16] Though it is possible in principle that the gauge non-singlet fields have larger value than unity along the $D$-term flat directions, it is unlikely in the chaotic configuration of the universe at the Planck-epoch since it requires a fine-tuning between various scalar fields.

[17] D.S. Salopek, Phys. Rev. Lett. 69, 3602 (1992).

[18] The idea to employ larger mass for the gravitino to solve various cosmological problems is due to J. Ellis, D.V. Nanopoulos, and M. Quiros, Phys. Lett. B174, 176 (1986).

[19] T.S. Bunch and P.C.W. Davies, Proc. R. Soc. London, A360, 117 (1978);

[20] A.D. Dolgov and D.P. Kirilova, Yad. Fiz. 51, 273 (1990) (English translation in Sov. J. Nucl. Phys. 51, 172 (1990)).

[21] If the scalar fields could grow beyond the Planck scale during the inflation, it might have caused another inflation, and only tiny density fluctuation of $O\left(10^{-15}\right)$ was possible. This discussion shows our model is free from this danger as well.

[22] L. Wolfenstein, Phys. Rev. D17, 2369 (1978); P. Mikheyev and A. Smirnov, Nuovo Cimento 9C, 17 (1986); H. Bethe, Phys. Rev. Lett. 56, 1305 (1986). 
[23] For a combined analysis of three experiments, see P. Anselmann et al, Phys. Lett. B 285, 390 (1992).

[24] T. Yanagida, in Proceedings of Workshop on the Unified Theory and the Baryon Number in the Universe, Tsukuba, Japan, 1979, edited by A. Sawada and A. Sugamoto (KEK, Tsukuba, 1979), p. 95; M. Gell-Mann, P. Ramond and R. Slansky, in Supergravity, proceedings of the Workshop, Stony Brook, New York, 1979, edited by P. Van Nieuwenhuizen and D.Z. Freedman (North-Holland, Amsterdam, 1979), p. 315.

[25] P. Langacker, PRINT-93-0127, February (1993).

[26] M. Fukugita and T. Yanagida, Phys. Lett. B 174, 45 (1986).

[27] V.A. Kuzmin, V.A. Rubakov, and M.E. Shaposhnikov, Phys. Lett. B 155, 508 (1985).

[28] P. Arnold and L. McLerran, Phys. Rev. D36, 581 (1987); S.Yu. Khlebnikov and M.E. Shaposhnikov, Nucl. Phys. B308, 885 (1988); J.S. Harvey and M.S. Turner, Phys. Rev. D42, 3344 (1990).

[29] Under the condition that $h_{33}$ does not blow up below the Planck scale, $m_{\nu_{\tau}}$ can be as large as $10 \mathrm{eV}$ with $\sin \beta=1$ and $M=10^{13} \mathrm{GeV}$.

[30] M. Davis, F.J. Summers, and D. Schlegel, Nature 359, 393 (1992); A.N. Taylor and M. Rowan-Robinson, Nature 359, 396 (1992).

[31] I. Affleck and M. Dine, Nucl. Phys. B249, 361 (1985). 


\section{FIGURE CAPTIONS}

Fig. 1 The potential Eq. (5) as a function of $\eta$, when $W_{i}=0$ for all the other fields.

Fig. 2 The value of $\eta$ at the minimum of the potential Eq. (5), as a function of $\left|W_{i}\right|^{2} /|W|^{2}$. 


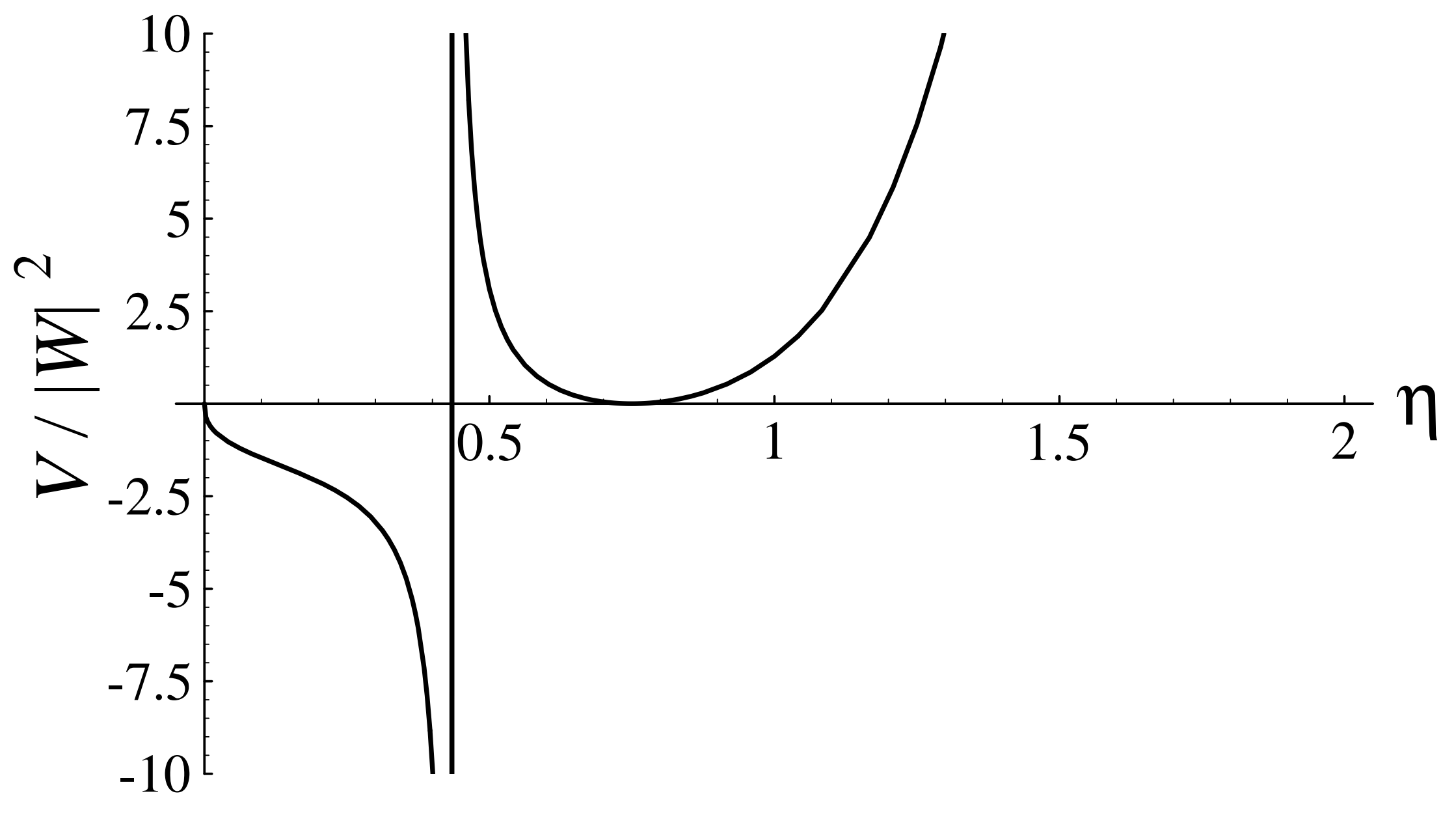

Fig. 1 
This figure "fig1-1.png" is available in "png" format from: http://arxiv.org/ps/hep-ph/9311326v1 
This figure "fig2-1.png" is available in "png" format from: http://arxiv.org/ps/hep-ph/9311326v1 


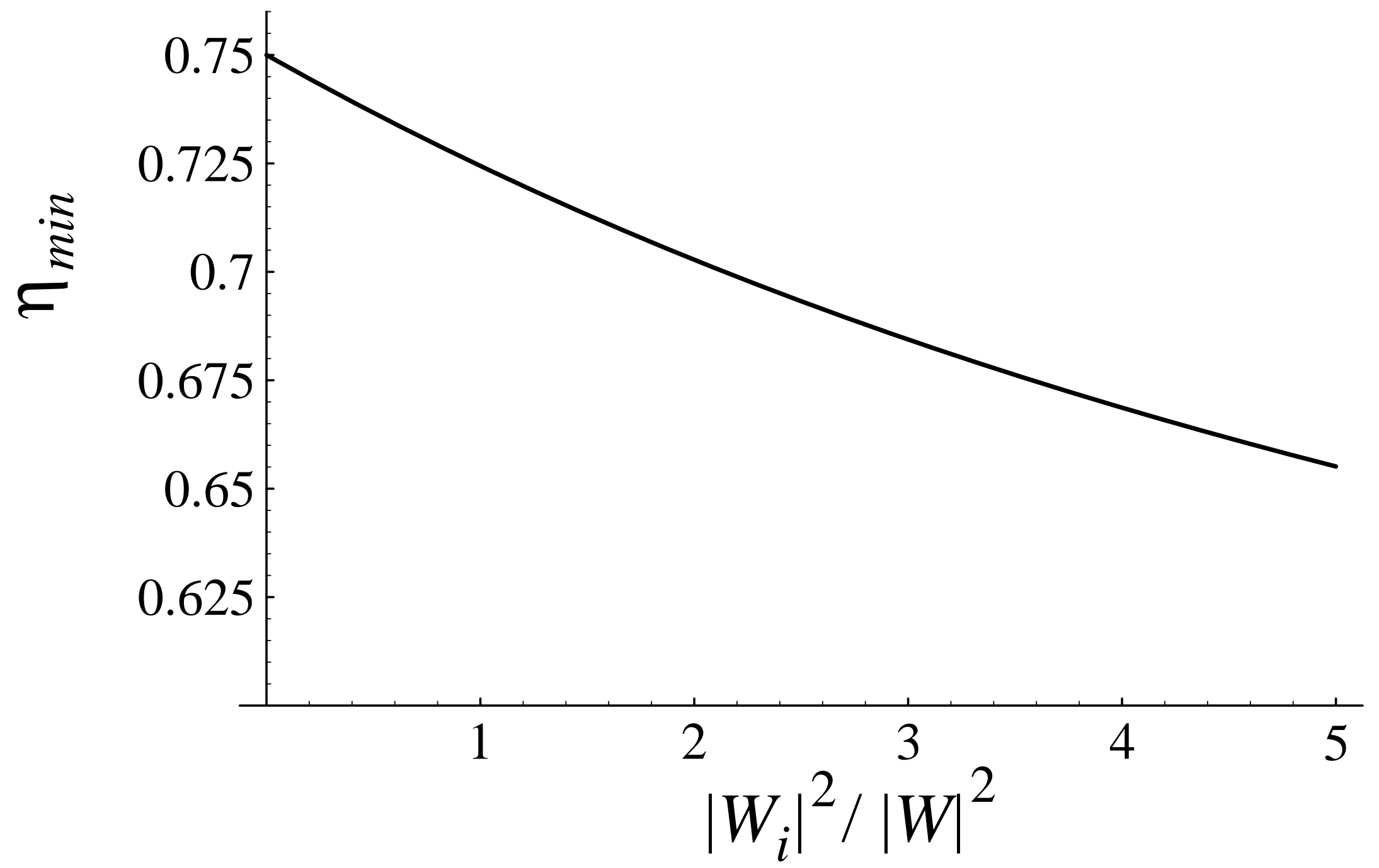

Fig. 2 